\title{
O BANCO DE SEMENTES DE UM TRECHO DE FLORESTA ATLÂNTICA MONTANA (SÃO PAULO, BRASIL)
}

\author{
BAIDER, C., ${ }^{1}$ TABARELLI, M. ${ }^{2}$ e MANTOVANI, W. ${ }^{1}$ \\ ${ }^{1}$ Departamento de Ecologia Geral, Universidade de São Paulo, C.P. 11461, CEP 05422-970, São Paulo, SP \\ ${ }^{2}$ Departamento de Botânica, Universidade Federal de Pernambuco, CEP 50670-901, Recife, PE \\ Correspondência para: Marcelo Tabarelli, Departamento de Botânica, CCB, Universidade Federal \\ de Pernambuco, Rua Moraes Rego, s/n, CEP 50670-901, Recife, PE \\ Recebido em 19/01/98 - Aceito em 17/06/98 - Distribuído em 30/06/99
}

(Com 1 figura)

\begin{abstract}
The soil seed bank of a tropical montane forest, Brazil

Soil seed bank of tropical forests is involved in the establishment of ecological groups and in woody species richness restoration during forest regeneration, after natural or antropic disturbances. In order to analyze the seed bank composition in a tropical montane forest, Southeastern Brazil, 57 soil samples were collected, inside a 2-hectare forest plot, representing $28,500 \mathrm{~cm}^{3}$ of collected soil. Along 17 months, 497 seeds germinated, $65 \%$ between 0 and $2.5 \mathrm{~cm}$ depth. They represented a density of 872 seeds $/ \mathrm{m}^{2}$. The seed bank analyzed is characterized as having two components: one composed by 66 herbaceous taxa, belonging to families with high richness of pioneer species; and another composed by 19 pioneer shrub and tree species, most of them with diaspores shorter than $1 \mathrm{~mm}$ length. The results found in the study site suggest that in the Atlantic montane forest soil seed bank can be important for the establishment of woody species, which form ecological groups involved in forest regeneration, following slash-and-burn. After treefall gaps, seed bank is probably responsible for the establishment of Melastomataceae species, the richest and more abundant group of pioneer species sampled in gaps.
\end{abstract}

Key words: soil seed bank, Atlantic forest, forest regeneration, pioneer species, Melastomataceae.

\section{RESUMO}

Durante a regeneração da floresta tropical, após perturbações naturais ou antrópicas, o banco de sementes do solo está envolvido no estabelecimento de grupos ecológicos e na restauração da riqueza de espécies lenhosas. Para a análise da composição de espécies do banco de sementes de um trecho de floresta Atlântica madura, no Sudeste do Brasil, foram coletadas 57 amostras de solo, distribuídas aleatoriamente em 2 ha, totalizando $28.500 \mathrm{~cm}^{3}$ de solo coletado. Durante um período de 17 meses germinaram 497 sementes, $65 \%$ distribuídas entre 0 e $2,5 \mathrm{~cm}$ de profundidade, totalizando $872 \mathrm{se}-$ mentes $/ \mathrm{m}^{2}$. O banco de sementes dessa floresta caracterizou-se pela presença de dois componentes: o herbáceo, composto por 66 morfotipos, pertencentes a famílias ricas em espécies pioneiras; e o arbustivo-arbóreo, formado por 19 espécies, em sua maioria, pioneiras com diásporos menores que 1 mm de comprimento. Os resultados obtidos sugerem que, na floresta Atlântica montana, o banco de sementes pode ter importância no estabelecimento de espécies de árvores e arbustos pioneiros, componentes de grupos ecológicos envolvidos na regeneração da floresta após corte e queima. Após a abertura de clareiras naturais, o banco de sementes pode ser o responsável pelo estabelecimento de espécies de Melastomataceae, principal grupo de árvores e arbustos pioneiros observados nas clareiras.

Palavras-chave: banco de sementes, espécies pioneiras, floresta Atlântica, Melastomataceae, regeneração. 


\section{INTRODUÇÃO}

O banco de sementes em florestas tropicais está envolvido em, pelo menos, quatro processos nos níveis de população e de comunidade. São estes: o estabelecimento de populações, a manutenção da diversidade de espécies, o estabelecimento de grupos ecológicos e a restauração da riqueza de espécies durante a regeneração da floresta após distúrbios naturais ou antrópicos (Harper, 1977; Solbrig, 1980; Uhl et al., 1988; Garwood, 1989).

A regeneração da floresta tropical ocorre após dois tipos básicos de distúrbio, que são o corte e queima das árvores e arbustos, com posterior utilização do solo para atividades agropastoris, e a abertura de clareiras naturais, causadas pela queda de uma ou mais árvores do dossel (GómezPompa et al., 1991).

Juntamente com a chuva de sementes, o banco de sementes é considerado um indicador do potencial de regeneração dessas florestas (Guevara \& Gómez-Pompa, 1972; Hopkins \& Graham, 1983; Epp, 1987; Garwood, 1989). Porém, nesses dois tipos de distúrbio, a importância relati- va de grupos de espécies e das fontes de diásporos para a regeneração é diferenciada. Em locais onde a floresta sofreu corte e queima, o estabelecimento de espécies pioneiras, a partir do banco de sementes, é considerado um fator regulador da velocidade de regeneração (Uhl, 1987; Whitmore, 1990). Putz \& Appanah (1987), na Malásia, e Lawton \& Putz (1988), no Panamá, sugerem que $85 \%$ da regeneração inicial dessas florestas estaria ligada ao banco de sementes.

Os bancos de plântulas e de jovens, que constituem a chamada regeneração avançada, parecem ter importância maior na regeneração da floresta após a abertura de clareiras pequenas $\left(<150 \mathrm{~m}^{2}\right)$, do que os diásporos externos, oriundos da chuva de sementes e daquelas presentes no estoque do solo. Na Venezuela, 95\% das árvores encontradas em clareiras pequenas, após 4 anos da sua formação, eram resultantes da regeneração avançada (Uhl et al., 1988).

O objetivo deste trabalho foi caracterizar o banco de sementes de um trecho de floresta Atlântica montana, discutindo sua importância no contexto de regeneração dessa floresta, após corte e queima e abertura de clareiras naturais.

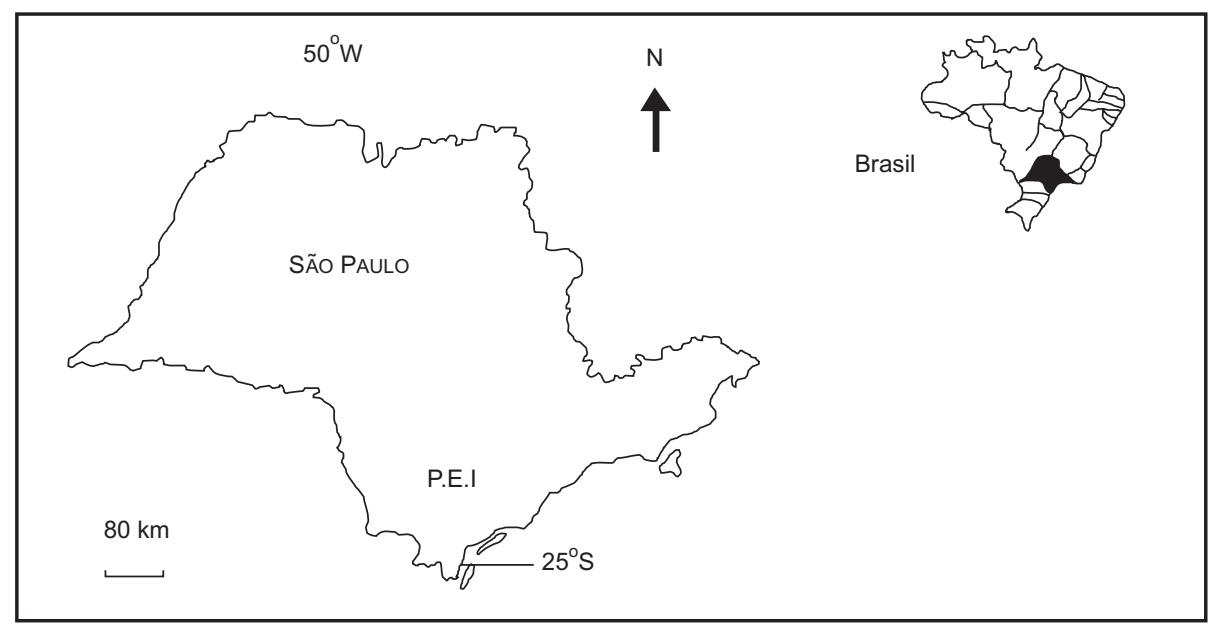

Fig. 1 - Mapa de localização do Parque Estadual Intervales (P.E.I.) no Estado de São Paulo. 


\section{MATERIAL E MÉTODOS}

\section{Área de estudo}

O estudo foi desenvolvido no Parque Estadual Intervales, situado na região sul do estado de São Paulo ( $24^{\circ} 2^{\prime}$ a $24^{\circ} 25^{\prime}$ 'S e $48^{\circ} 03^{\prime}$ a $\left.48^{\circ} 30^{\prime} \mathrm{W}\right)$, próximo ao município de Ribeirão Grande, ocupando uma área de 38.536 ha (Fig. 1). Essa unidade de conservação dispõe-se, predominantemente, sobre a Serra de Paranapiacaba, em altitudes que variam de $60 \mathrm{~m}$ a $1.100 \mathrm{~m}$ de altitude (Almeida, 1974). Há o predomínio de solos do complexo Campos do Jordão, às vezes associados a solos argilosos, como Litossolos Vermelho e Amarelo e Podzólicos Vermelho-Amarelo, muito ácidos e pobres em bases trocáveis (Comissão de Solos, 1960; Almeida, 1974; Baider, 1994).

O clima local é classificado como Cfa de Köppen, sendo uma região de transição climática entre o clima tropical quente das latitudes baixas e o temperado mesotérmico das latitudes médias, com influência da altitude (Baider, 1994). Há, pelo menos, um mês com temperatura inferior a $18^{\circ} \mathrm{C}$ e a temperatura média anual é inferior à $22^{\circ} \mathrm{C}$, podendo ocorrer de um a quatro eventos de geada a cada ano. Embora ocorra variação na distribuição das chuvas ao longo do ano, não há período de seca, sendo a precipitação média anual em torno de $1.720 \mathrm{~mm}$ (Leme, 1997). A vegetação que recobre a região pode ser classificada, de acordo com a altitude, como floresta ombrófila densa submontana ou montana, formações que compõem a floresta Atlântica brasileira (Veloso et al., 1991). Informações sobre a composição florística dessa floresta no local de estudo podem ser obtidas em Feitosa do Nascimento (1994).

\section{Banco de sementes}

Com base em informações dos moradores locais, foi selecionado um trecho de 2 ha de floresta montana madura a $910 \mathrm{~m}$ de altitude, a qual estava circundada por florestas em regeneração. $\mathrm{O}$ trecho selecionado apresentava características fisionômicas similares às de outros de floresta Atlântica montana descritos na literatura (veja Mantovani et al., 1990; Tabarelli, 1997).

A análise do banco de sementes foi realizada através do método de germinação (Brown, 1992). Foram coletadas 57 amostras ao acaso, dentro da área de 2 ha, nas profundidades de 0 a $2,5 \mathrm{~cm}$ e de 2,5 a $5,0 \mathrm{~cm}$, desprezando-se a serapilheira dura.
O material foi coletado com auxílio de um anel de metal de $11,3 \mathrm{~cm}$ de diâmetro e 2,5 cm de altura, resultando em um volume total de solo coletado de $28.500 \mathrm{~cm}^{3}$ e em uma área de $0,57 \mathrm{~m}^{2}$. Essas amostras foram colocadas em vasos plásticos, dispostos em mesas recobertas com 3 telas diferentes: uma com náilon branco, uma com sombrite $30 \%$ e uma com sombrite $80 \%$, com o objetivo de estimular a germinação do maior número de espécies.

Os vasos eram regados periodicamente. $\mathrm{O}$ sombreamento real produzido pelas telas, medido com um luxímetro, foi de $27 \%$ no náilon branco, $41,5 \%$ com sombrite $30 \%$ e $86 \%$ com sombrite $80 \%$. O ensaio foi montado na cidade de São Paulo, local de clima equivalente ao da área de estudo.

Foram colocados, aleatoriamente, 8 vasos com areia em cada mesa para controle da contaminação do ensaio pela chuva local de sementes. As plântulas emergentes foram retiradas periodicamente, entre 30 e 60 dias de idade, quando atingiam tamanhos que permitissem identificá-las. Para a contagem e retirada das plântulas foi necessário tirar a cobertura de sombrite. O solo do vaso era revolvido quando não havia recrutamento por 2 meses. $\mathrm{O}$ ensaio permaneceu montado no período de janeiro de 1992 a maio de 1993 (17 meses).

As espécies arbóreo-arbustivas identificadas foram classificadas em relação às suas estratégias de regeneração: a) pioneiras e b) tolerantes à sombra; e de dispersão: a) zoocóricas e b) anemocóricas, conforme Pijl (1982) e Hartshorn (1978). Os diásporos das espécies identificadas foram classificados em duas classes de tamanho: (1) diásporos menores que $1 \mathrm{~mm}$ de comprimento e (2) diásporos entre 1 e $5 \mathrm{~mm}$. Para a classificação das espécies e dos diásporos foram utilizadas informações publicadas na Flora Ilustrada Catarinense (Reitz, 1965), em Van Roosmalen (1985) e no conhecimento dos autores sobre a biologia das mesmas.

\section{RESULTADOS}

Considerando todos os níveis de sombreamento, durante o período de 17 meses, germinaram 497 sementes, resultando em uma densidade de 872 sementes $/ \mathrm{m}^{2}$.

Entre as 497 sementes que germinaram, $65 \%$ estavam entre 0 e $2,5 \mathrm{~cm}$ e as demais (35\%) entre 2,5 e $5 \mathrm{~cm}$. 
Duzentos e setenta e cinco sementes deram origem a 66 morfotipos herbáceos, distribuídos, preferencialmente, entre 0 e $2,5 \mathrm{~cm}$ de profundidade, resultando em uma densidade de 483 sementes $/ \mathrm{m}^{2}$. Duzentas e vinte e duas sementes deram origem a 19 espécies arbóreo-arbustivas, das quais $64,4 \%$ ocorreram entre 0 e $2,5 \mathrm{~cm}$ de profundidade, resultando em uma densidade de 389 sementes $/ \mathrm{m}^{2}$ (Tabela 1 ).

Entre os morfotipos herbáceos estavam presentes aqueles pertencentes a Compositae, Gramineae, Rubiaceae, Malvaceae, Solanaceae e Cruciferae, entre outras. Considerando apenas as espécies arbustivoarbóreas, Melastomataceae foi a família com maior porcentagem de espécies $(52,6 \%)$ e de indivíduos $(88,2 \%)$. Moraceae e Rubiaceae participaram com $5,2 \%$ das espécies e com $0,9 \%$ e $0,4 \%$ dos indivíduos.

Myrsinaceae e Solanaceae representaram $10,2 \%$ das espécies e $2,2 \%$ e 5,4\% dos indivíduos, respectivamente, enquanto Euphorbiaceae representou $15,7 \%$ das espécies e $2,7 \%$ dos indivíduos. Dos 17 táxons arbóreo-arbustivos identificados em nível de espécie, $82,3 \%$ foram classificados como pioneiros e $17,7 \%$, como tolerantes à sombra. $\mathrm{O}$ grupo das pioneiras foi o mais abundante, pois representou $98 \%$ dos indivíduos amostrados.

A maioria das espécies apresentou síndrome de zoocoria $(94,1 \%)$, com predominância de diásporos menores que $1 \mathrm{~mm}$ de comprimento $(52,9 \%)$ (Tabela 2).

\section{DISCUSSÃO}

\section{Banco de sementes}

O banco de sementes dessa floresta caracterizou-se pela presença de dois componentes: o herbáceo, composto de morfotipos pertencentes a famílias ricas em espécies pioneiras; e o arbustivo-arbóreo, com predominância de arbustos pioneiros, com diásporos menores que $1 \mathrm{~mm}$, ambos distribuídos, preferencialmente, até $2,5 \mathrm{~cm}$ de profundidade (Tabelas 1 e 2).

Symington (1933 apud Whitmore, 1990) foi quem realizou o primeiro trabalho com banco de sementes em uma região tropical, observando a germinação de espécies provenientes de solos de florestas maduras em Kepong, na Malásia.

O experimento foi refeito por Keay (1960 apud Whitmore, 1990), na Nigéria, e depois em outras regiões tropicais, sempre com o mesmo resultado: o banco de sementes das florestas tropicais é constituído, basicamente, por espécies pioneiras herbáceas e arbustivo-arbóreas de ciclo de vida curto (veja Enright, 1985; Fetcher et al., 1987; Putz \& Appanah, 1987; Smith, 1987; Saulei \& Swaine, 1988).

Conforme Hopkins \& Graham (1984) e Vásquez-Yanes \& Orozco-Segovia (1987), espécies herbáceas pioneiras não são componentes das florestas tropicais, mas aparecem em grande número no banco de sementes, pois, geralmente, apresentam dormência facultativa, além de possuírem mecanismos eficientes de dispersão. Por exemplo, Putz \& Appanah (1987), na Malásia, encontraram um banco com 131 sementes $/ \mathrm{m}^{2}$, sendo $31 \%$ dos indivíduos oriundos de espécies herbáceas, $40 \%$, de espécies arbustivas e 39\%, de espécies arbóreas.

Espécies de hábito arbóreo, como as grandes pioneiras $A$. triplinervia, $P$. glabrata e $H$. alchornioides, encontradas no local de estudo, são comuns nos bancos de sementes. Em um número elevado de estudos em florestas tropicais, espécies arbóreas têm sido encontradas, em especial, pioneiras e grandes pioneiras (Guevara \& GómezPompa, 1972; Hall \& Swaine, 1980; Enright, 1985; Lawton \& Putz, 1988; Hopkins et al., 1990), representando entre $18 \%$ e $91 \%$ do total de espécies encontradas nos bancos (Garwood, 1989).

Entre as espécies arbóreo-arbustivas amostradas no local de estudo, a síndrome de dispersão dominante foi a zoocoria. Nas florestas neotropicais ocorrem riquezas elevadas de árvores e arbustos pioneiros zoocóricos, como aquelas dos gêneros Vismia (Guttiferae), Piper (Piperaceae), Clusia (Clusiaceae), Psidium (Myrtaceae), Rapanea, Miconia, Leandra, Solanum e Cecropia (Cecropiaceae) (Whtimore, 1990; Gómez-Pompa et al., 1991; Tabarelli, 1997).

Em sua maioria, apresentam diásporos pequenos ( $<5 \mathrm{~mm}$ de comprimento), dispersos por frugívoros generalistas, como os passeriformes das famílias Pipridae e Emberizidae, nos casos de Piper, Miconia, Leandra e Rapanea; por morcegos frugívoros no caso de Solanum; por frugívoros especialistas das famílias Cotingidae, Cracidae, Ramphastidae e Cebidae, nos casos de Cecropia e Psidium (Van Roosmalen, 1985; Stiles \& Rosselli, 1993; Sick, 1997). 
TABELA 1

Totais de plântulas (N), densidade de sementes $\left(\mathrm{sem} / \mathrm{m}^{2}\right)$ e número de espécies e morfotipos (S), recrutados em diferentes profundidades no banco de sementes de um trecho de floresta Atlântica.

\begin{tabular}{|c|c|c|c|c|c|c|c|c|}
\hline $\begin{array}{c}\text { Prof. } \\
\mathbf{( c m )}\end{array}$ & $\begin{array}{c}\mathbf{N} \\
\text { total }\end{array}$ & $\mathbf{s e m} / \mathbf{m}^{\mathbf{2}}$ & $\begin{array}{c}\mathbf{N} \\
\text { herbáceas }\end{array}$ & $\mathbf{S}$ & $\mathbf{s e m} / \mathbf{m}^{\mathbf{2}}$ & $\begin{array}{c}\mathbf{N} \\
\text { arbustivo-arbóreas }\end{array}$ & $\mathbf{S}$ & $\mathbf{s e m} / \mathbf{m}^{\mathbf{2}}$ \\
\hline $0-2,5$ & 323 & 567 & 180 & - & 316 & 143 & - & 251 \\
\hline $2,5-5,0$ & 174 & 305 & 95 & - & 167 & 79 & - & 138 \\
\hline Total & 497 & 872 & 275 & 66 & 483 & & 222 & 19 \\
\hline
\end{tabular}

TABELA 2

Espécies arbustivo-arbóreas com seus respectivos números de indivíduos (N), hábitos, estratégias de regeneração $(\mathrm{P}=$ pioneira, $\mathrm{T}=$ tolerante à sombra $)$, síndromes de dispersão $(\mathrm{A}=$ anemocoria, $\mathrm{Z}=$ zoocoria) e classes de tamanho de diásporos $(A=<1 \mathrm{~mm} ; \mathrm{B}=>1 \mathrm{e}<5 \mathrm{~mm})$ recrutadas no banco de sementes de um trecho de floresta Atlântica.

\begin{tabular}{|c|c|c|c|c|c|}
\hline Família/espécie & $\mathbf{N}$ & Háb.* & Reg. & Disp. & Dias. \\
\hline \multicolumn{6}{|l|}{ Euphorbiaceae } \\
\hline Alchornea glandulosa Poeppig & 3 & Árvore & $\mathrm{P}$ & Z & B \\
\hline Hyeronima alchornioides Fr. Allem. & 2 & Árvore & $\mathrm{P}$ & Z & B \\
\hline Pera glabrata (Schott.) Baill & 1 & Árvore & $\mathrm{P}$ & Z & B \\
\hline \multicolumn{6}{|l|}{ Melastomataceae } \\
\hline Leandra aff. alterninervia Cogn. & 23 & Arbusto & $\mathrm{P}$ & Z & A \\
\hline Leandra hirtella Cogn. & 2 & Arbusto & $\mathrm{P}$ & Z & A \\
\hline Leandra mosenii Cogn. & 53 & Arbusto & $\mathrm{P}$ & Z & A \\
\hline Leandra niangaeformis Cogn. & 18 & Arbusto & $\mathrm{P}$ & Z & A \\
\hline Leandra aff. purpurascens Cogn. & 36 & Arbusto & $\mathrm{P}$ & Z & A \\
\hline Miconia pyrifolia Naod. & 2 & Arbusto & $\mathrm{P}$ & Z & A \\
\hline Miconia theaezans (Bompl.) Cogn. & 41 & Arbusto & $\mathrm{P}$ & Z & A \\
\hline Tibouchina mutabilis Cogn. & 10 & Árvore & $\mathrm{P}$ & A & A \\
\hline sp 1 & 3 & - & - & - & - \\
\hline sp 2 & 8 & - & - & - & - \\
\hline \multicolumn{6}{|l|}{ Moraceae } \\
\hline Ficus aff. luschnatiana Miq. & 2 & Árvore & $\mathrm{T}$ & Z & A \\
\hline \multicolumn{6}{|l|}{ Myrsinaceae } \\
\hline Cybianthus brasiliensis (Mez.) Agost. & 1 & Árvore & $\mathrm{T}$ & Z & $\mathrm{B}$ \\
\hline Rapanea ferruginea (Ruiz et Pav.) Mez. & 4 & Árvore & $\mathrm{P}$ & Z & B \\
\hline \multicolumn{6}{|l|}{ Rubiaceae } \\
\hline Psychotria stachyoides Benth. & 1 & Arbusto & $\mathrm{T}$ & Z & $\mathrm{B}$ \\
\hline \multicolumn{6}{|l|}{ Solanaceae } \\
\hline Solanum erianthum D. Don & 10 & Árvore & $\mathrm{P}$ & Z & $\mathrm{B}$ \\
\hline Solanum inaequale Vell. & 2 & Árvore & $\mathrm{P}$ & Z & B \\
\hline Total & 222 & & & & \\
\hline
\end{tabular}

*Árvore $=$ indivíduos $\oplus 10 \mathrm{~cm}$ dap; arbustos $=$ indivíduos $<10 \mathrm{~cm}$ dap. 
Espécies que ocorrem enterradas no banco de sementes, via de regra, são pequenas. Entre as vantagens adaptativas, comumente conferidas a essa característica, estão a menor pressão de predação e a maior facilidade de incorporação ao estoque do solo (Orozco-Segovia, et al. 1993). Na verdade, raramente encontram-se sementes maiores que $5 \mathrm{~mm}$ nos bancos (Foster, 1986), apesar de, no local de estudo, alguns diásporos maiores, como os de Ocotea catharinensis (Lauraceae) e de Hymenaea courbaril (Leguminosae), terem sido facilmente visíveis na serapilheira.

Conforme Orozco-Segovia et al., (1993), essa ausência reflete a viabilidade pequena de grandes sementes, além de outros fatores limitantes, como a pressão de predação acentuada, a maior vulnerabilidade ao ataque de fungos, além da maior dificuldade física para o seu enterramento.

A densidade de sementes encontrada no trecho de floresta estudado (Tabela 1) foi superior às obtidas em outras florestas tropicais, apesar de não se observar em florestas tropicais montanas a mesma riqueza e dominância de pioneiras, observadas nas florestas tropicais de terras baixas (Tabarelli \& Mantovani, 1997). As maiores densidades de indivíduos encontradas em banco de sementes ocorrem em florestas secundárias ou fazendas antigas. Em florestas tropicais e temperadas maduras há, em média, menos de 500 sementes $/ \mathrm{m}^{2}$, que são, quase exclusivamente, de espécies ausentes ou raras na vegetação local, provindas de diferentes locais e épocas (Garwood, 1989). A elevada densidade observada no local de estudo pode ser conseqüência do histórico de perturbação dessa floresta, na qual se observam, ainda hoje, pequenas áreas cobertas por vegetação herbáceoarbustiva, circundando os trechos de floresta madura. Nessas áreas são comuns espécies herbáceas de Compositae, Rubiaceae, Gramineae, Malvaceae e Solanaceae, entre outras (Baider, 1994; Feitosa do Nascimento, 1994).

Em relação à distribuição das sementes dentro do perfil do solo, esta constitui um indicativo do comportamento pretérito da chuva e do banco de sementes e do potencial de estabelecimento de algumas espécies após perturbações, como escorregamentos e desenraizamentos de árvores de grande porte. No local de estudo, $35 \%$ das sementes que germinaram foram encontradas entre 2,5 e 5 $\mathrm{cm}$ de profundidade, evidenciando a oferta pre- térita de sementes e o potencial de estabelecimento de espécies herbáceas e arbustivo-arbóreas, especialmente nos caso de revolvimento do solo (Lawton \& Putz, 1988). Em outras florestas tropicais e em diferentes áreas de floresta Atlântica, também tem sido encontrado um elevado número de sementes até a profundidade de $5 \mathrm{~cm}$ (veja Roizman, 1993).

Os resultados encontrados nesse trecho de floresta Atlântica montana corroboram a hipótese de que os bancos de sementes encontrados nos solos tropicais são, na sua maioria, constituídos por ervas, arbustos e árvores pioneiras, que produzem sementes pequenas, enterradas, preferencialmente, até $2,5 \mathrm{~cm}$ de profundidade. Esse padrão emerge, apesar das grandes diferenças de composição de espécies e de estratégias de dispersão observadas nas florestas tropicais (veja Gentry, 1983, 1988a).

\section{Importância para regeneração}

A importância do banco de sementes para a regeneração das florestas tropicais relaciona-se ao estabelecimento de grupos ecológicos, como o das pioneiras, e com a restauração da riqueza de espécies arbóreo-arbustivas. A regeneração da floresta Atlântica montana, após corte e queima, caracteriza-se não só pela substituição de espécies pioneiras por tolerantes à sombra, como também pela substituição direcional de formas de crescimento e de histórias de vida, a partir de espécies herbáceas, dominando, consecutivamente, arbustos, árvores pioneiras de ciclo de vida curto e de ciclo longo (Tabarelli, 1997). Conforme Uhl (1987), a sucessão da floresta tropical, após este tipo de distúrbio, pode ser melhor descrita pelo modelo de facilitação (senso Connell \& Slatyer, 1977). Espécies pioneiras invadem lentamente um sítio disponível à colonização e facilitam o estabelecimento de outras, pois agem como abrigo para os vetores de dispersão, melhoram as condições de fertilidade do solo e fornecem hábitats adequados ao recrutamento. Dessa forma, espécies de ervas, arbustos, árvores pioneiras de ciclo de vida curto e longo constituem grupos ecológicos com funções distintas na regeneração da floresta.

A regeneração da floresta Atlântica no Sul e Sudeste do Brasil pode ser descrita por uma seqüência de estádios dominados por espécies de Andropogon (Gramineae), Baccharis (Compositae), 
Rapanea, Miconia e Alchornea (Klein, 1980); por Imperata (Gramineae), Baccharis, Tibouchina e Alchornea (Tabarelli, 1997) ou por Imperata, Baccharis, Tibouchina e Nectandra (Lauraceae), cronosseqüência observada na área de estudo (Feitosa de Nascimento, 1994). Essas espécies compõem as comunidades pioneiras, as quais, no caso das arbóreas, parecem imprescindíveis ao estabelecimento de árvores e arbustos tolerantes à sombra que compõem a floresta madura (Gómez-Pompa \& Vázquez-Yanes, 1981). A análise da composição do banco de sementes (Tabela 2) revela que este contém, pelo menos, espécies de três grupos ecológicos envolvidos na regeneração da floresta após corte e queima. São estas: os arbustos pioneiros dos gêneros Leandra e Miconia, as árvores pioneiras de ciclo curto de Solanum, Rapanea e Tibouchina e as grandes pioneiras dos gêneros Alchornea, Hyeronima e Pera.

Além desse fato, em estudos de banco de sementes, em outras comunidades florestais brasileiras, observaram-se espécies de Trema, Cecropia e Solanum (Daniel \& Jankauskis, 1989; Roizman, 1993; Takahasi \& Moura, 1994; Tabanez \& Viana, 1994). Na floresta Atlântica, espécies desses gêneros ocorrem como subdominantes nos estádios iniciais de regeneração (Mantovani, 1993; Tabarelli, 1997), evidenciando o possível papel do banco de sementes no estabelecimento de grupos ecológicos envolvidos na regeneração da floresta. Especificamente na floresta Atlântica montana, a origem da chuva de sementes, formadora do banco descrito, tem sua origem na ocorrência de populações de pioneiras estabelecidas em hábitats iluminados desta floresta, como clareiras naturais, topos de morros e orla de rios (Falkenberg \& Voltolini, 1993; Lima \& Guedes-Bruni, 1997; Tabarelli, 1997).

No que se refere à restauração da riqueza de espécies, durante a regeneração da floresta, o banco de sementes parece ter um papel restrito, pois é constituído, principalmente, de arbustos pioneiros de Melastomataceae. Florestas neotropicais apresentam entre 118 e 306 espécies de árvores/ha $(\oplus 10 \mathrm{~cm}$ dap) (Gentry, 1988b; Valencia et al., 1994), as quais representam, aproximadamente, $50 \%$ da riqueza das espécies lenhosas (Gentry \& Emmons, 1987). Em um trecho de floresta Atlântica montana, em São Paulo, Tabarelli \& Mantovani (1999) encontraram 136 espécies de árvores. Gran- de parte da riqueza de árvores e arbustos dessa floresta pertence a Myrtaceae, Lauraceae, Sapotaceae e Monimiaceae, desde os estádios iniciais de regeneração (Tabarelli et al., 1993; Leitão Filho, 1993). São espécies com sementes entre $5 \mathrm{~mm}$ e $30 \mathrm{~mm}$ de comprimento, ausentes do banco de sementes. Como declarado por Roberts (1981 apud Saulei \& Swaine, 1988), o banco de sementes das florestas tropicais é composto, na maioria, de sementes ausentes ou raras da vegetação local, não sendo um mecanismo importante para o estabelecimento de espécies da floresta madura, principal componente da riqueza de árvores e de arbustos nestas florestas.

Na regeneração da floresta após a abertura de clareiras, os bancos de plântulas e de jovens, que constituem a chamada regeneração avançada, parecem ter importância maior do que os diásporos externos, oriundos da chuva de sementes e das sementes presentes no estoque do solo (Uhl et al., 1981; Denslow, 1987). Em clareiras pequenas, o banco de sementes deve ter um papel ainda menor na sua colonização, pois as condições microclimáticas podem não mudar de maneira significativa, de forma que propiciem a germinação das sementes presentes no solo (Lawton \& Putz, 1988). $\mathrm{Na}$ Venezuela, 95\% das árvores encontradas em clareiras pequenas, após 4 anos da sua formação, eram resultantes da regeneração avançada (Uhl et al., 1988).

Em algumas florestas tropicais, são exceções as grandes clareiras formadas pelo desenraizamento de grandes árvores, nas quais têm sido observada a dominância ou densidades elevadas de espécies pioneiras (Swaine \& Hall, 1983; Brokaw, 1985). Tabarelli (1997) analisou a colonização de 30 clareiras naturais em um trecho de floresta Atlântica montana, em São Paulo, onde foram amostradas 220 espécies de árvores e arbustos. Entre estas, 53 (24\%) foram árvores e arbustos pioneiros, sendo seis espécies $(11,3 \%)$ classificadas como grandes pioneiras e as demais $(88,7 \%)$ como pioneiras de ciclo de vida curto. Quinze espécies de Miconia, 9 de Leandra e 2 de Rapanea constituíram o principal grupo de pioneiras nessa floresta, pois representaram $49 \%$ do total das espécies e $52,1 \%$ do total dos indivíduos pioneiros amostrados.

Esses resultados sugerem que o banco de sementes é um mecanismo importante no estabe- 
lecimento de espécies de Melastomataceae, principal grupo de pioneiras amostrados por Tabarelli (1997). Porém, em nenhuma das clareiras amostradas pelo autor as espécies pioneiras foram dominantes ou o grupo mais rico, ou seja, não há evidências de que o estabelecimento desse grupo de espécies seja um condição necessária para o estabelecimento de outros grupos, ou contribua para a restauração da riqueza de espécies durante a regeneração da floresta.

Os resultados obtidos nesse estudo, aliados às informações sobre a regeneração da floresta Atlântica montana, constituem evidências de que o banco de sementes pode ser importante no estabelecimento de espécies de árvores e de arbustos pioneiros, constituintes de grupos ecológicos envolvidos na regeneração após corte e queima da floresta. Após a abertura de clareiras naturais, o banco de sementes pode ser responsável pelo estabelecimento de espécies de Melastomataceae, principal grupo de árvores e arbustos pioneiros observados na colonização de clareiras. Estudos quantificando a dependência de sementes enterradas no solo para o estabelecimento dos diferentes grupos de pioneiras envolvidos na regeneração da floresta são necessários para avaliar a real contribuição do banco nesse processo.

\section{REFERÊNCIAS BIBLIOGRÁFICAS}

ALMEIDA, F. F. M., 1974, Fundamentos geológicos do relevo paulista. Instituto de Geologia e Geografia, Série Teses e Monografias 14, São Paulo.

BAIDER, C., 1994, O banco de sementes e de plântulas na sucessão da Mata Atlântica. Dissertação de Mestrado, Instituto de Biociências, Universidade de São Paulo, São Paulo.

BROKAW, N. V. L., 1985, Gap-phase regeneration in a tropical forest. Ecology, 66: 682-687.

BROWN, D., 1992, Estimating the composition of a forest seed bank: a comparison of the seed extraction and seedling emergence methods. Can. J. Bot., 70: 16031612.

COMISSÃO DE SOLOS, 1960, Levantamento de reconhecimento dos solos do Estado de São Paulo. Bol. Serv. Nac. Pesq. Agron., 12: 1-634.

CONNELL, J. H. \& SLATYER, R. O., 1977, Mechanisms of succession in natural communities and their role in community stability and organization. Am. Nat., 111: $1119 / 1140$.

DANIEL, O. \& JANKAUSKIS, J., 1989, Avaliação da metodologia para o estoque de sementes do solo em floresta de Terra Firme na Amazônia brasileira. IPEF, 41/42: 18-36.
DENSLOW, J. S., 1987, Tropical rain forest gaps and tree species diversity. Annu. Rev. Ecol. Syst., 18: 431-451.

ENRIGHT, N., 1985, Evidence of a soil seed bank under rain forest in New Guinea, Melbourne. Austr. J. Ecol., 10: $67-$ 71.

EPP, G. A., 1987, The seed bank of Eupatorium odoratum along successional gradient in a tropical rain forest in Ghana. J. Trop. Ecol., 3: 139-149.

FALKENBERG, D. B. \& VOLTOLINI, J. C, 1993, The montane cloud forest in the Southern Brazil. In: L. S. Hamilton, O. J. Jovir \& F. N. Scatena (eds.), Tropical Montane Cloud Forest Symposium, Puerto Rico, Proceedings 1: 86-93.

FEITOSA DO NASCIMENTO, F. A., 1994, A sucessão secundária inicial na mata atlântica, sobre a Serra de Paranapiacaba, Ribeirão Grande, SP. Dissertação de Mestrado, Instituto de Biociências, Universidade de São Paulo, São Paulo.

FETCHER, N., OBERBAUER, S. F., ROJAS, G. \& STRAIN, B. R., 1987, Efectos del régimen de luz sobre la fotossíntesis y el crescimento en plántulas de árboles de um bosque lluioso tropical de Costa Rica. Rev. Biol. Trop., 35: $97-110$.

FOSTER, S. A., 1986, On adaptative value of large seeds for tropical moist forest trees: a review and synthesis. Bot. Rev., 52: 260-269.

GARWOOD, N. C., 1989, Tropical soil seed banks: a review, pp. 149-209. In: M. A. Leck, V. T. Parker \& R. L. Simpson (eds.), Ecology of soil seed banks. Academic Press, San Diego.

GENTRY, A. H., 1983, Dispersal ecology and diversity in neotropical forest communities. Sonderb. Naturwiss. Rev. Hamburg, 7: 303-314.

GENTRY, A. H., 1988a, Changes in plant community diversity and floristic composition on environmental and geographical gradients. Ann. Missouri Bot. Gar., 75: 1-34.

GENTRY, A. H., 1988b, Tree species richness of upper Amazonian forests. Proc. Natl. Acad. Sci., 85: 156-159.

GENTRY, A. H. \& EMMONS, L. H., 1987, Geographical variation in fertility, phenology, and composition of the understory of neotropical forests. Biotropica, 19: 216-227.

GÓMEZ-POMPA, A. \& VÁZQUEZ-YANES, C. N., 1981, Successional studies of a rain forest in Mexico, pp. 247 266. In: D. C. West, H. H. Schugart \& D. B. Botkin (eds.), Forest concepts and application, Springer-Verlag, New York.

GÓMEZ-POMPA, A., WHITMORE, T. C. \& HADLEY, M., 1991, Tropical rain forest: regeneration and management. Blackwell, New York.

GUEVARA, S. S. \& GÓMEZ-POMPA, A., 1972, Seeds from surface soils in a tropical region of Veracruz, Mexico. $J$. Arnold. Arb., 53: 312-335.

HALL, J. B. \& SWAINE, M. D., 1980, Seed stocks in Ghanian forests soils. Biotropica, 12: 256-263.

HARPER, J. L., 1977, Population biology of plants. Academic Press, London. 
HARTSHORN, G. S., 1978, Treefalls and tropical forest dynamics, pp. 617-638. In: P. B. Tomlinson, \& M. H. Zimmermman (eds.), Tropical trees as living systems, Cambridge Univ. Press, New York.

HOPKINS, M. S. \& GRAHAM, A. W., 1983, The composition of soil seed banks beneath lowland tropical rainforests in North Queensland, Australia. Biotropica, 15: 90-99.

HOPKINS, M. S. \& GRAHAM, A. W., 1984, Viable soil seed banks in disturbed lowland tropical rainforests in North Queensland, Australia, Melboure. Austr. J. Ecol., 9: 7179.

HOPKINS, M. S.,TRACEY, J. G. \& GRAHAM, A. W., 1990 The size and composition of soil seed banks in remnant patches of three structural rainforest types in North Queensland, Australia, Melboure. Austr. J. Ecol., 15: 43-50.

KLEIN, R. M., 1980, Ecologia da flora e vegetação do Vale do Itajaí. Sellowia, 32: 165-389.

LAWTON, R. O. \& PUTZ, F. E., 1988, Natural disturbance and gap-phase regeneration in wind-exposed tropical cloud forest. Ecology, 69: 764-777.

LEITÃO FILHO, H. F. (org.), 1993, Ecologia da Mata Atlântica em Cubatão. Editora da UNESP e Editora da Universidade de Campinas, Campinas.

LEME, A. S., 1997, Relações entre padrões de forrageio, morfologia e uso de recursos no gênero Drymophila (Aves: THAMNOPHILIDAE). Dissertação de Mestrado, Instituto de Biociências, Universidade de São Paulo, São Paulo.

LIMA, H. C. \& GUEDES-BRUNI, R. R., 1997, Diversidade de plantas vasculares na Reserva Ecológica de Macaé de Cima, pp. 29-40. In: H. C. de Lima \& R. R. GuedesBruni (eds.), Serra de Macaé de Cima: diversidade florística e conservação em Mata Atlântica, Jardim Botânico do Rio de Janeiro, Rio de Janeiro.

MANTOVANI, W., 1993, Estrutura e dinâmica da floresta Atlântica na Juréia, Iguape, SP. Tese de Livre-docência, Instituto de Biociências, Universidade de São Paulo, São Paulo.

MANTOVANI, W., RODRIGUES, R. R., ROSSI, L., ROMANIUC-NETO, S., CATHARINO, E. L. M. \& CORDEIRO, I., 1990, A vegetação na Serra do Mar em Salesópolis. In: ACIESP (ed.), II Simpósio Ecossistemas da Costa Sul e Sudeste: estrutura, composição e manejo, São Paulo, Anais, 2: 348-384.

OROZCO-SEGOVIA, A., SANCHEZ-CORONADO, M. E. \& VÁZQUEZ-YANES, C., 1993, Light environment and phytochrome - controlled germination in Piper auritum. Funct. Ecol., 7: 585-590.

PIJL, V. D., 1982, Principles of dispersal in higher plants. 3a ed., Springer-Verlag, Berlim.

PUTZ, F. E. \& APPANAH, S., 1987, Buried seeds, newly dispersed seeds, and dynamics of a lowland forest in Malaysia. Biotropica, 19: 326-333.

REITZ, R. (ed.), 1965, Flora ilustrada catarinense. Herbário Barbosa Rodrigues, Itajaí, SC, 149v.
ROIZMAN, L. G., 1993, Fitossociologia e dinâmica do banco de sementes de populações arbóreas de floresta secundária em São Paulo. Dissertação de Mestrado, Instituto de Biociências, São Paulo, SP.

SAULEI, S. M. \& SWAINE, M. D., 1988, Rain forest seed dynamics during succession at Gogol, Papua-Nova Guiné. J. Ecol., 62: 675-719.

SICK, H., 1997, Ornitologia brasileira, uma introdução. Editora Nova Fronteira, Rio de Janeiro.

SMITH, A. P., 1987, Respuestas de hierbas del sotobosque tropical a claros ocasionados por la caída de árboles. Rev. Biol. Trop., 35 (suplemento 1): 111-118.

SOLBRIG, O. T., 1980, Demography and evolution in plant populations. University of California Press, Berkeley.

STILES, F. G. \& ROSSELLI, L., 1993, Consumption of fruits of the Melastomataceae: how diffuse is coevolution. Vegetatio, 107/108: 57-73.

SWAINE, M. D. \& HALL, J. B., 1983, Early succession on cleared forest land in Ghana. J. Ecol., 71: 601-627.

TABANEZ, A. J. \& VIANA, V. M., 1994, Efeito de borda sobre o banco de sementes em um fragmento florestal de Piracicaba, SP. In: Sociedade Brasileira de Botânica (ed.), XLV Congresso Nacional de Botânica, São Leopoldo, RS, Resumos, 1: 161.

TABARELLI, M., 1997, A regeneração da floresta Atlântica montana. Tese de Doutorado, Instituto de Biociências, Universidade de São Paulo, São Paulo.

TABARELLI, M. \& W. MANTOVANI., 1997, Colonização de clareiras naturais na floresta Atlântica no sudeste do Brasil. Rev. Brasil. Bot., 20: 57-66.

TABARELLI, M. \& MANTOVANI, W., 1999, A riqueza da floresta Atlântica de encosta no estado de São Paulo (Brasil). Rev. Brasil. Bot. (aceito).

TABARELLI, M. VILLANI, J. P. \& MANTOVANI, W., 1993, Aspectos da sucessão secundária no Parque Estadual da Serra do Mar, SP. Rev. Inst. Ftal, 5: 101-114.

TAKAHASI, A. \& MOURA, L. C., 1994, Levantamento fitossociológico associado a um estudo preliminar sobre banco de sementes do solo de uma comunidade secundária em Rio Claro, SP. In: Sociedade Brasileira de Botânica (ed.), XLV Congresso Nacional de Botânica, São Leopoldo, RS, Resumos, 1: 10.

UHL, C., 1987, Factors controlling succession following slash-and-burn agriculture in Amazonia. J. Ecol., 75: 377-407.

UHL, C., CLARK, K., CLARK, H. \& MURPHY, P., 1981, Early plant succession after cutting and burning in the upper Rio Negro region of the Amazonian Basin. J. Ecol., 69: 631-649.

UHL, C., CLARK, K. \& MAQUIRINO, P., 1988, Vegetation dynamics in Amazonian treefall gaps. Ecology, 69: 751763.

VALENCIA, R., BALSLEV, H. \& PAZ Y MIÑO, G., 1994, High tree alpha-diversity in Amazonian Ecuador. Biodiver. Conser., 3: 21-28. 
VAN ROOSMALEN, G. M., 1985, Fruits of the Guianan flora. Institute of Systematic Botany, Utrecht University, Holanda.

VÁZQUEZ-YANES, C. \& OROZCO-SEGOVIA, A., 1987, Fisiologia ecologica de semillas en la Estacion de Biologia Tropical "Los Tuxtlas", Veracruz, Mexico. Rev. Biol. Trop., 35 (suplemento 1): 85-89.
VELOSO, H. P., RANGEL FILHO, A. L. R. \& LIMA, J. C. A., 1991, Classificação da vegetação brasileira, adaptada a um sistema universal. IBGE, Rio de Janeiro.

WHITMORE, T. C., 1990, An introduction to tropical rain forests. Blackwell, London. 